\title{
A Giant Caseous Calcification of the Mitral Annulus with Uncommon Presentation
}

\section{Valentina Boasi*}

Medical Doctor, Sanremo Hospital, ASL1 Imperiese, Italy

Submission: March 24, 2021; Published: April 20, 2021

*Corresponding author: Valentina Boasi, Medical Doctor, Sanremo Hospital, ASL1 Imperiese, Via Giovanni Borea 56, Sanremo, Imperia, Italy

\section{Case Report}

We described the case of a 73 years old woman who underwent an abdomen CT for a renal problem and a giant cardiac calcification was detected. The patient bad no cardiac symptoms except for occasional palpitations. The ECG showed sinusal rhythm with right bundle block.
The echocardiography showed a mild mitral regurgitation without stenosis and a not well defined "enlargement of the mitral anulus and of myocardium in the posterior inter-ventricular sept" with an increased hyperechogenicity but not a clear calcification.

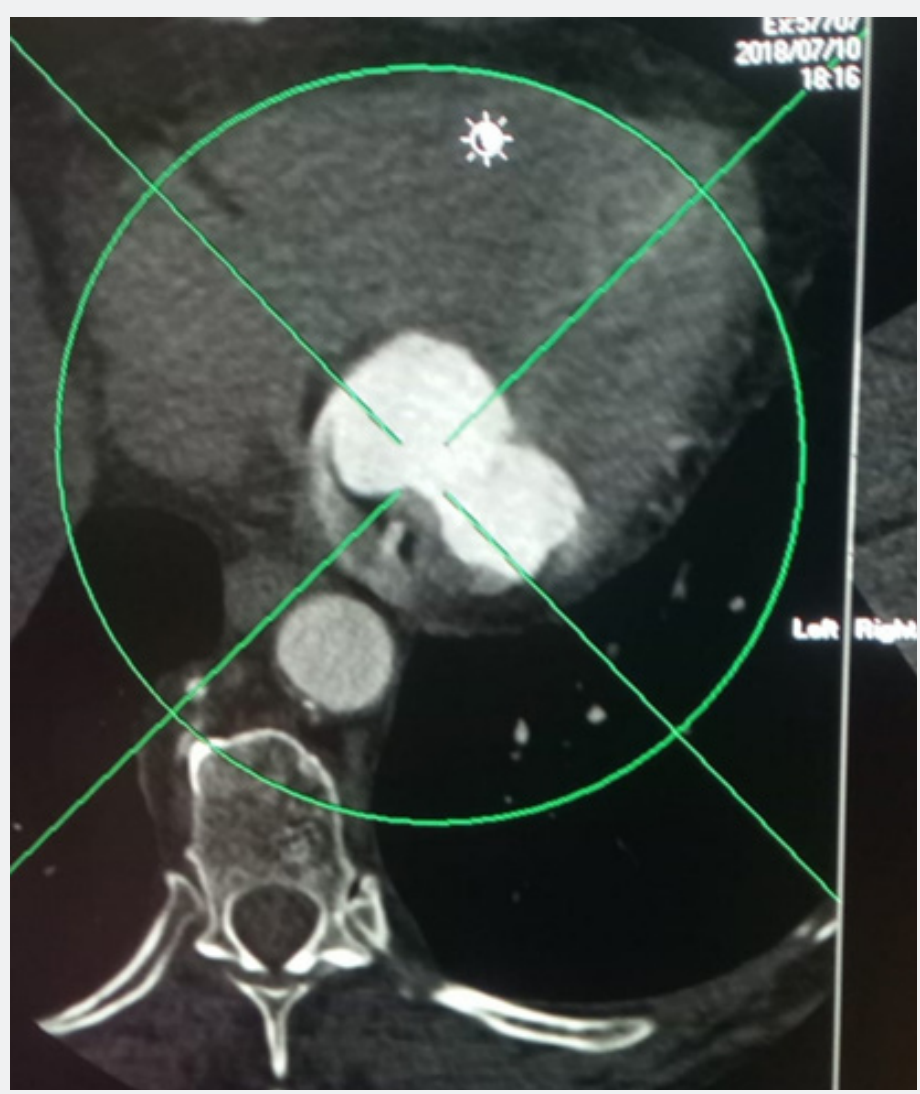

Figure 1: 
Patient underwent a cardiac CT with and without contrast medium that revealed a hyperdense mass located at the anterior and posterior mitral ring, highly suggestive of caseous calcification of the mitral annulus (see Figures 1 and 2). The mass extended circumferentially for about $75 \mathrm{~mm}$ with a maximum thickness of $19 \mathrm{~mm}$. In contrast with other cases of mitral calcification there was not an important involvement of valve apparatus. It showed also coronary calcifications with a stenosis around $50 \%$ in the second segment of the anterior descendant artery. The patient was asymptomatic for angor or dyspnea, so she was prescribed an ECG-holter and treated conservatively and followed with echocardiography.

Despite its benign prognosis this uncommon finding generated a lot of anxiety for the patient until the correct diagnosis was formulated because it could mimics different diseases and cardiologist and radiologist should become used to recognize it.

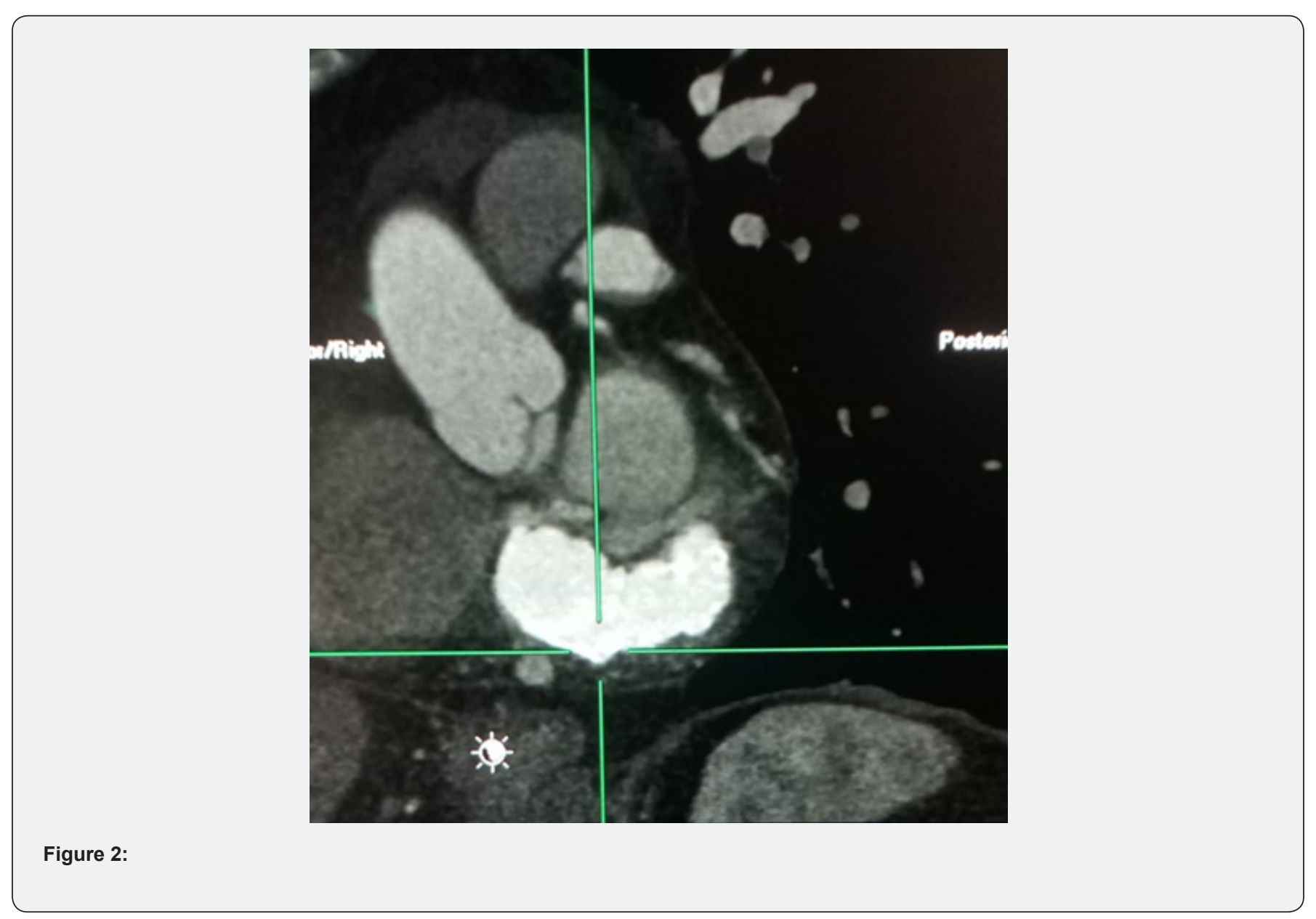

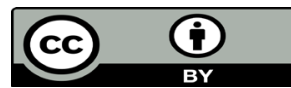

This work is licensed under Creative Commons Attribution 4.0 License DOI: 10.19080/JOCCT.2021.16.555950

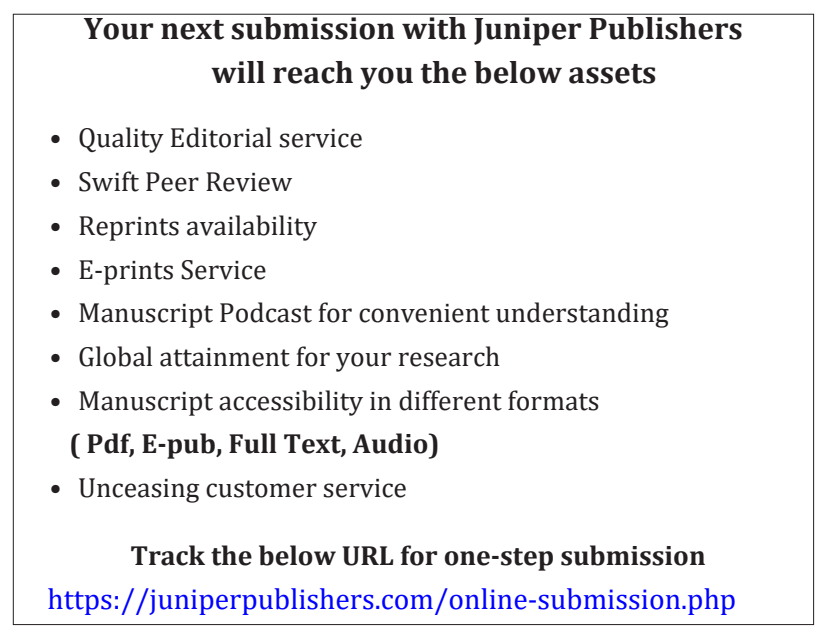

